\title{
Competition in the Built Environment: Scaling Laws for Cities, Neighbourhoods and Buildings
}

\author{
Michael Batty ${ }^{1}$
}

Published online: 18 August 2015

(C) Kim Williams Books, Turin 2015

\begin{abstract}
Built environments at any spatial scale are represented as sets of like objects-cities, neighbourhoods, buildings but also components such as streets, parks, etc.,- - which are distributed spatially according to certain rules that we are only just beginning to detect and measure. Unlike normally distributed attributes of the population, these urban elements often scale according to forces that determine large numbers of small elements and a small number of large ones. While many objects evolve from small to large, not all objects can be large as resources as well as physical limits determine the distribution of their sizes. The theory behind such size distributions is referred to as scaling and the shape of such distributions is quite well-defined by various power and exponential laws. As objects grow and evolve, or are even designed, their form changes qualitatively due to the forces of competition and the constraints on space. Here we explore these ideas for cities, neighbourhoods in towns, and buildings, specifically high buildings, revealing that there are both important differences as well as similarities in their form, function, and structure.
\end{abstract}

Keywords Scaling $\cdot$ Power laws $\cdot$ Random growth $\cdot$ City populations $\cdot$ Rank size $\cdot$ Building heights

\section{Self-Organisation, Scale and Size}

Lionel March in his introduction to the special issue of the Nexus Network Journal on shape grammars (vol. 13, no. 1, 2011) begins his reflections on architecture and mathematics by saying:

Michael Batty

m.batty@ucl.ac.uk

1 Centre for Advanced Spatial Analysis (CASA), University College London, 90 Tottenham Court Road, London W1T 4TJ, UK 
I used to quote Lord Kelvin: 'When you can measure what you are speaking about and express it in numbers, you know something about it, but when you cannot express it in numbers, your knowledge is of a meagre and unsatisfactory kind' But what numbers? (March 2011: 6).

So Lionel March articulated the challenge that has lived with us for the last half century or more. We have painfully explored ways in which we might define our understanding of cities formally using algebra and measure, but only in the last decade are we beginning to find universal properties that can be measured numerically and which define 'the kind of problem that a city is' (Jacobs 1961: 428 et. seq.). The search for universals is of course the quest of physics but slowly we are getting glimpses of such universality in social systems through signatures which define order in geometry, as for example in fractals, in information theory, and in qualitative transformations encapsulated in allometry and scaling. Indeed scaling - the ability to transform a given set of objects from one scale, spatial and/or temporal, to another without changing its structure-provides a fascinating entry point to thinking about properties of cities and buildings that define the elements of the urban system. In this essay, we will pursue this quest to find simple order in sets of objects which scale spatially, thus providing some sense in which systems such as cities, which tend to evolve more naturally than buildings (which tend to be designed), change as they grow, expand, decline or contract. We are but at the beginning of this quest and what follows is inevitably speculative. But this is a direction in which to expand architecture through its form and function using mathematics, and bears considerable promise for our understanding of complex systems.

Many processes of growth and evolution begin with the objects in their species being created small with their development path being one of growth to some equilibrium or optimal size that then perpetuates through regeneration, and proceeds ultimately to their demise through death. Few natural populations of biological origin depart from these processes of birth, regeneration, and death although for artificial objects of our own creation, there is nothing intrinsic in their composition that provides a theoretical limit on their longevity. In studying the built environment, the notion of naturally growing or evolving artefacts has replaced the idea that all the objects of our creation are imposed on us by top-down design, as it has become increasingly clear that the sort of competitive processes that characterise 'survival-of-the-fittest' play a role in the way the built environment evolves. This can be seen to a greater extent in all the objects that we create and design, from cities to neighbourhoods to buildings, as well as other elements that make up their physical form, such as infrastructure, materials, and building components.

One signature of an evolving competitive process is the size distribution of the objects that are created. Components of the built environment at different scales are distributed with many small objects and few large. Unlike attributes of human beings that tend to be normally distributed around some mean such as size (height/ weight), cities themselves, their neighbourhood sizes, and the heights of their buildings all show much bigger numbers of small objects than large but with the largest objects themselves dominating the size distribution. Over time, it has been observed that although this distribution changes in that all objects begin small on 
their way to bigness, objects move up and down the size hierarchy but with the overall distribution remaining highly stable in macro terms over time. In fact, one can be quite precise about the shape that such distributions approximate. If we look at the frequency of occurrence $f(x)$ by size $x$, the distribution approximates an inverse function similar to a rectangular hyperbola which we can define as $f(x) \sim x^{-\alpha}$. The rectangular hyperbola is a special case where $\alpha=1$ although as we shall see, we are never able to define such functions with such precision. A large body of literature suggests that some sort of inverse power function is a good approximation to many distributions that evolve from small to large that we find in nature and society (Clauset et al. 2009). A demonstration of the difference between this kind of distribution and the normal, which is equally if not more important in charactering the distribution of human attributes, is shown in Fig. 1.

In fact, we must be clear that when we talk of the typical distribution of components in the built environment as being approximated by an inverse power, this suggests that the underlying distribution is something different. If we examine for example the number of cities against their size, then the number of the very smallest cities (hamlets, for example) tends to be smaller than the next size of settlements up (villages, say) and as we carry on to the next size class up, at some point the frequency will peak and then begin to decline monotonically with size according to something that can be approximated by an inverse power function. This suggests that the distribution of sizes is really lognormal but over most of its length, it can be approximated by a power law. This would mean that in Fig. 1, where we show these two distributions, if we compressed the first half of the normal distribution to the left of its mode, it would become a lognormal and in fact, theoretical arguments which we note in the next section, indeed suggest that the lognormal is the most appropriate (Simon 1955).

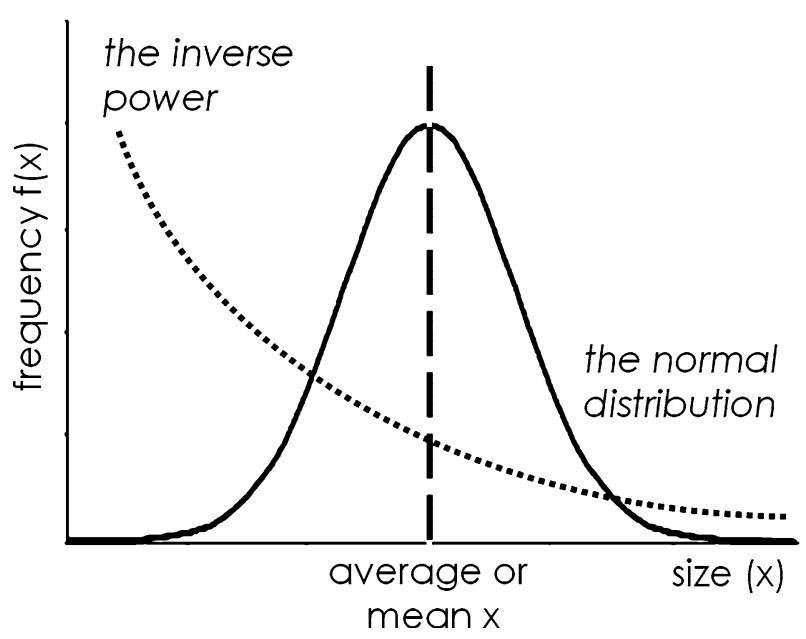

Fig. 1 The normal and inverse power distributions. The normal has a mean but the power does not; the power can also be seen as an approximation to the right tail of a lognormal distribution which visually is a normal distribution with its mode skewed to the left of the normal 
It is quite easy to see intuitively why we might have a smaller number of big objects rather than a larger number, especially if objects grow in size. When resources are fixed, not every object can become big, and in a fixed space, which is tantamount to saying that there are fixed resources, distributions which are sustainable are those which follow some sort of inverse distribution between frequency and size. If it takes a certain level of resources to either grow or indeed design an object, then in general it takes longer to design a bigger object and thus there are likely to be fewer of them. We can give this argument real power if the objects relate to one another through interactions. The number of interactions between two small objects is proportionately much smaller than between two objects which are, say double the size of the smaller. If we have two towns of 1000 persons each, then there are $1000^{2}=1 \mathrm{~m}$ possible interactions, while if the towns are double the size at 2000 persons, there are $2000^{2}=4 \mathrm{~m}$ possible interactions, so doubling the size produces four times the number of links. In growing systems, this doubling suggests that in a constrained space, there are proportionately fewer large than small objects.

To an extent this is competition in the raw. One might object that in urbanism and architecture, when we design objects we have no such competitive constraints in mind, but in fact a little reflection shows we do. The number of objects of a different size depends on the resources we have to build them, and if we go back one stage to the economy and take the example of residential buildings, it is clear that there are many more poor people than rich. In fact the distribution of income follows an inverse power law or lognormal, as many from Vilfredo Pareto in the late nineteenth century onwards have observed. This must be reflected in the sizes of houses that we build and can afford. Taking the argument one stage further, this is reflected in the size of any kind of facility that we use. In turn this is a function of the frequency of use, which in turn depends on the resources we can mobilise to build them. As our wealth and income increases, one might expect the size of things that we can build to get larger but their distribution may well remain stable. In fact in general such distributions do remain stable although the positions of their objects by size within this envelope may well vary quite radically over time.

\section{Baseline Nihilism: Creation and Destruction of Built Environments}

We will develop the simplest possible model that generates a baseline distribution which we can use as a comparator. Imagine a series of individuals, each with a small but identical level of resources which they can use to sustain themselves. Now, the only way an individual can change the resources they have is by drawing a lottery ticket, the value of which tells them how much additional resource they will gain. Note that for this hypothetical example, an individual's resources will always increase. The lottery is truly random, so the individuals have no control over what they get and in the long term, their resources are entirely dictated by this random process. Now the best way to see what happens is to look at a sequence of increments to wealth for an individual. If the individual starts with $r_{i}(1)$ resources at time $t=1$, then with a probability of $\rho_{i}(1)$, the individual's resources will increase 
to $r_{i}(2)=r_{i}(1)\left(1+\rho_{i}(1)\right)$. These resources will then increase to $r_{i}(3)=r_{i}(2)(1+-$ $\left.\rho_{i}(2)\right)=r_{i}(1)\left(1+\rho_{i}(1)\right)\left(1+\rho_{i}(2)\right)$ and in general the level of resources at time $t$ will equal $r_{i}(t)=r_{i}(1) \prod_{\tau=1}^{t}\left(1+\rho_{i}(\tau)\right)$. Now the probability of choosing a lottery ticket of highest value is random, so the choice of a sequence of high probabilities becomes increasingly unlikely. If we consider a large population of individuals, the distribution of wealth after a large number of draws will be highly skewed to the smallest levels, following something like a power law. We will not prove this to be the case but there are plenty of demonstrations in the literature (Clauset et al. 2009). It is worth noting that if the increment of wealth can be a decrement, when we impose the condition that the total resource for each individual cannot fall below zero, the power law also emerges. In short we are really looking at a model which generates the right hand side of the lognormal distribution, which of course can be approximated by an inverse exponential or power law (Blank and Solomon 2000).

We refer to this way of looking at the evolution of a size distribution as nihilist. By this we mean that it is devoid of any theory about how cities and their development are constituted other than as a collection of elements that evolve or are designed. Of course, in context this process only mirrors what actually happens when we ground it in the fact that resources are always limited, but it is easy to see from this description that a random process can lead to the desired results. It is in fact the process of choice that dictates the selection of sizes. The stochastic process we have outlined needs to be modified as suggested in (Blank and Solomon 2000) amongst others. We need to allow an individual's resources-or the size of the relevant object - to decrease as well as increase in size, and thus our probability also needs to be moderated by a random event that takes resources away as well increases them. If we do this, then what we require is a lower limit-a threshold below which the size of the object cannot fall (it cannot go negative in size); if the object does touch this limit, it remains there until the next draw is positive. In terms of stochastic processes, we call this a random walk with a reflecting barrier. This means that objects can grow and decline within the distribution. This process then mirrors certain features of well-known size distributions. Cities, for example, follow an inverse power distribution but some cities move up and down in the process while others remain at a fixed position for long periods. New York City for example has been number 1 in size since records began in the United States in the 1790 Census. But if one looks at the top fifty cities in $450 \mathrm{BCE}$, then none of these is now in the top fifty some 2500 years later (Batty 2006). In contrast of course, very few skyscrapers which have been around since the late nineteenth century have been demolished as yet - though of course in time they will-while the dynamics of those kinds of building processes are very different from those pertaining to the evolution of cities. We will look at this later.

In this sense then, we can see the process of building and development as one of creation and destruction following economic processes first elucidated by Joseph Schumpeter (1939) with respect to technological change. Schumpeter argued that in the case of capital development, perfectly serviceable and economically valuable assets were continually destroyed as soon as new technologies came on the scene. This is quite easy to see in terms of urban development, and the only reason why skyscrapers have been so long lasting in places like New York City is because of the 
difficulties of destroying their structure and creating new buildings on their site as well as the quality of their construction. In fact, in places like Hong Kong, the process of destroying high buildings is proceeding apace as use value changes. This is dependent upon land use too, but as building technologies improve with respect not only to construction but demolition, the half-life of objects comprising the built environment, such as tall buildings, is likely to decrease. When it comes to cities, however, there is much greater change as cities grow and decline, mainly without disappearing. Going back to the example cited earlier, while New York City has been number one in the US for the last 220 years, several cities which were high on the list in 1790 have disappeared from the top 100, such as Savannah, Georgia, and Richmond, Virginia. In fact, although many of the components that make up the built environment do reveal clear scaling, the processes that generate them are distinctly different, as are the temporal dynamics of their evolution and design.

\section{City Size Distributions}

Before we proceed to examine different distributions, we need to change the way in which we present and explore such frequencies. So far we have simply suggested that the frequency defining a set of objects by size is likely to follow a lognormal distribution where most of the distribution can be approximated by an inverse power function (or even a negative exponential which is a suitable transform). However there is a much cleaner way of presenting such a system which is to rank the objects by their size and examine this distribution. In fact this is related to the cumulative distribution and we refer to this following (Zipf 1949) as the rank-size distribution. We derive this as follows. First write the basic frequency $f\left(x_{i}\right)$ of the size $x_{i}$ of object $i$ in inverse power form as

$$
f(x) \sim x^{-\alpha}
$$

where $\alpha$ is the parameter of the distribution. Then if we accumulate the frequencies as $F(x)$ by integrating (1) with respect to $x$, we get

$$
F(x) \sim \frac{x^{1-\alpha}}{1-\alpha} .
$$

and we can now write the counter-cumulative distribution function in terms of the cumulative frequency as

$$
x \sim(1-\alpha) F(x)^{\frac{1}{1-\alpha}} \sim r^{-\beta} .
$$

The rank is defined as $r$ and the parameter as $\beta$, which, as we will see, is related to the degree of competition-the inequalities between the objects that compose the size distribution. There are some limits on the value of $\alpha$ and $\beta$ as noted in (Newman 2005). The distribution is degenerate if $\alpha=1$ and it is thus suggested that the lower bound on this parameter is greater than 1. $\beta$ is thus a measure of the deviation from degeneracy, which would mean deviation from the situation where all elements are of the same size. Therefore the greater the value of $\beta$, the more unequal the 
distribution and by implication, the greater the competition between the various elements of the distribution.

\section{City Systems and Rank-Size Dynamics}

\section{Global Cities}

We will begin by examining city size distributions at a global level and then look at those for a continental land mass, the United States. Globally, city sizes reflect the organisation of the world's economic trade system as well as political entities. It might be remarked that there is unevenness in this system as different economic blocks display different levels of development. We have taken our data by defining the top 533 cities which have populations greater than one million persons as of 1 April, as compiled in Brinkhoff's City Population data base (http://www. citypopulation.de/world/Agglomerations.html). In our analysis, it is very clear that at the scale of city populations, there are considerable difficulties in defining what a city is. The problem is well-known and we will illustrate this first by comparing Brinkhoff's data with a similar data set derived from UN data 5 years earlier, in 2010. This data records all cities greater than 750,000 persons, of which there were 589. It comes from the Nordpil's processing of the UN Population Division data at every 5 years from 1950 and is available at https://nordpil.com/ resources/world-database-of-large-cities/. The profiles are similar but by no means identical. When we linearise the rank-size equation by taking logarithms of Eq. (3), we fit the equation
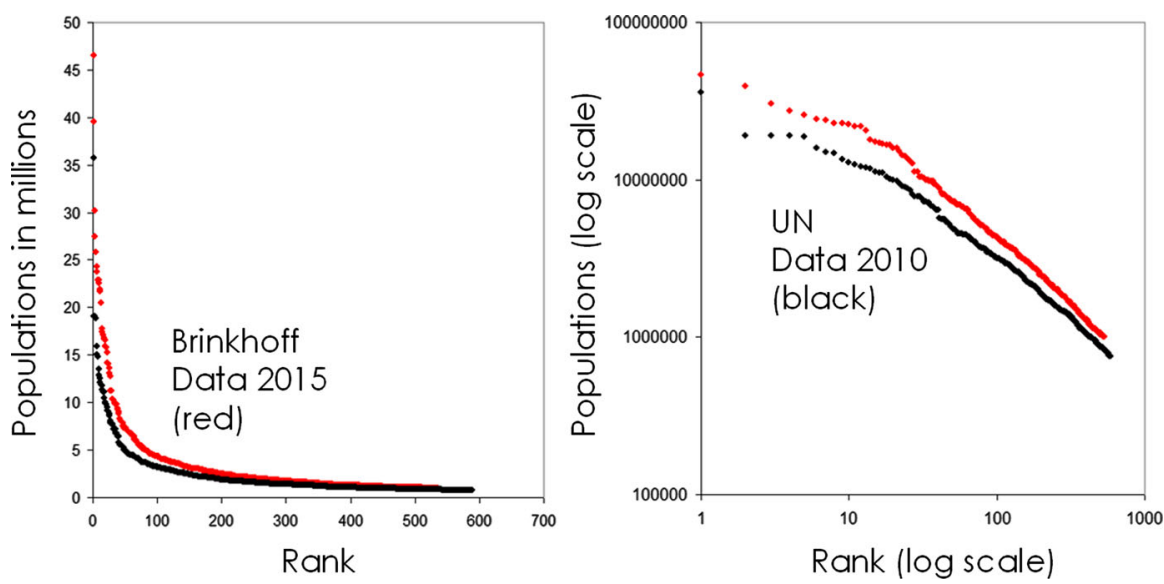

Fig. 2 Raw and logarithmic rank plots of two world city data distributions. a Brinkhoff data (left); b UN data (right) 


$$
\log x=K-\beta \log r
$$

to the Brinkhoff data where we estimate $\beta=0.782$, while the for UN data $\beta=0.717$. The proportion of the variance accounted for by these estimations is very close at $R^{2}=0.982$ and $R^{2}=0.984$ respectively, but the data sets, despite these close estimates, are quite different at one level. In the top ten city sizes for each data set, only five are common: the largest in the Brinkhoff data set is Guanzhou with a population of $46 \mathrm{~m}$, but this city does not even appear in the top ten of the UN data set. Shanghai is twice the size in the Brinkhoff data, and this reveals how difficult it is to define large cities. The problem of course becomes easier as the scale grows finer (that is, gets smaller from global to local).

We show the data for these two global systems in Fig. 2, where we have plotted their raw form in Fig. (2a) and their logarithmic form in Fig. (2b). Essentially, when we examine the largest objects in their class or group, we immediately run into the problem of artificially bounding the system. In Fig. 2, we can see the effect of this along with other differences between the distributions. The fact that the 2010 data lines are somewhat 'flatter' than the 2015 lines in the plots is not accounted for merely by differences in the volumetric measures. Of course, world populations have grown between these two dates, but if we take the top 500 from each set, we get 1.349 billion for the UN data at 2010 and 1.876 billion for the Brinkhoff data. Clearly the definition of urban cores is very different between each data set with the cities generally in the Brinkhoff data being greater in size and area than the UN. In fact Fig. 2 implies as much but several features worth noting add to this difficulty.

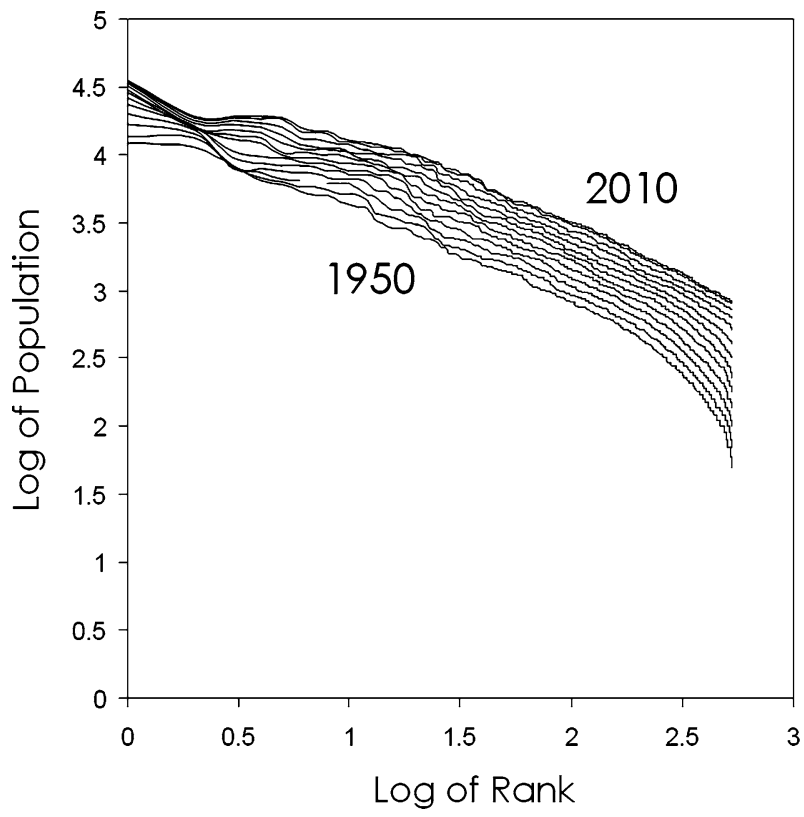

Fig. 3 Rank-size plots for global city data from 1950 to 2010 
While Fig. 2a shows that the cut-off is quite artificial, Fig. 2b shows how different are the relative slopes of the rank-size curves. At the very top end of the distributions which contain the largest cities, these curves typically seem to fall off. The slopes are conditioned by these largest estimates, and at the top end of the UN data the slope is much flatter. But clearly all this depends on how much data we collect and how far we are able to collect data on city size. At some point, statistics no longer count populations as being in towns and cities and it is clear that any complete analysis must attempt to collect as much data as possible so that some semblance of completeness is made for this kind of analysis.

Before we examine the form of some really large data sets of city sizes, we will look again at the UN data, which we have available over time for all cities with a population greater than 750,000 from 1950 to 2010, at five-year intervals. We have excluded cities which were greater than 100,000 in 1950 but had not reached 750,000 in 2010. We show these size distributions in Fig. 3.

The effect of the cut-off is very clearly seen. Indeed the characteristic increasing slope of the distributions as they cover smaller and smaller cities, in effect reveals an increase in competition at smaller city size levels. In fact the 1950 curve almost reveals the classic lognormal signature of the rank-plot which we will show in the next section. At the top end of these distributions, there is greater volatility and it is there that we see the effect of compression amongst the largest city sizes. We also can see in some city systems (but not in these plots), dramatic increases in the very largest city sizes, called the primate city or dragon-king effect (Pisarenko and Sornette 2012). To take these ideas further, we now need to move to finer scales and look at the distribution of cities in continental contexts-here for the United States and Britain.

\section{National City Systems: At Continental Levels and Below}

When we move to the scale of cities as being part of continents or nations, then the dynamics of competition changes. In fact, as we move to finer scales, in general we hypothesise that the degree of competition measured by the value of the scaling parameter falls, with the spatial objects comprising the system becoming more even. This is largely because larger objects can merge into one another and their size and spacing is finer than at the global level. We will demonstrate this for two city systems: for the United States which is a continental land mass as well as a distinct nation, and for Britain (excluding Ireland), it too being a distinct national territory but of much smaller scale. The first data set on city sizes for the United States is based on the number of incorporated places (which in 2000 was 19,452) available from the US Census from 1970. We show the rank-size plots for each decade from 1970 to 2000 in Fig. 4, where the complete data is an inset and the heavy tail of the distribution for the top 10,000 places is the main graphic. It is immediately clear what happens to the plot when we cut off over half the places with the lowest populations. The original plot departs dramatically from the linearised power law revealing, as in the inset, the characteristic signature of ranked city sizes, which is quite clearly lognormal. In other words, we lose much of the dynamics of city size distributions when we simply deal with the heavy or upper tail of the distribution. 


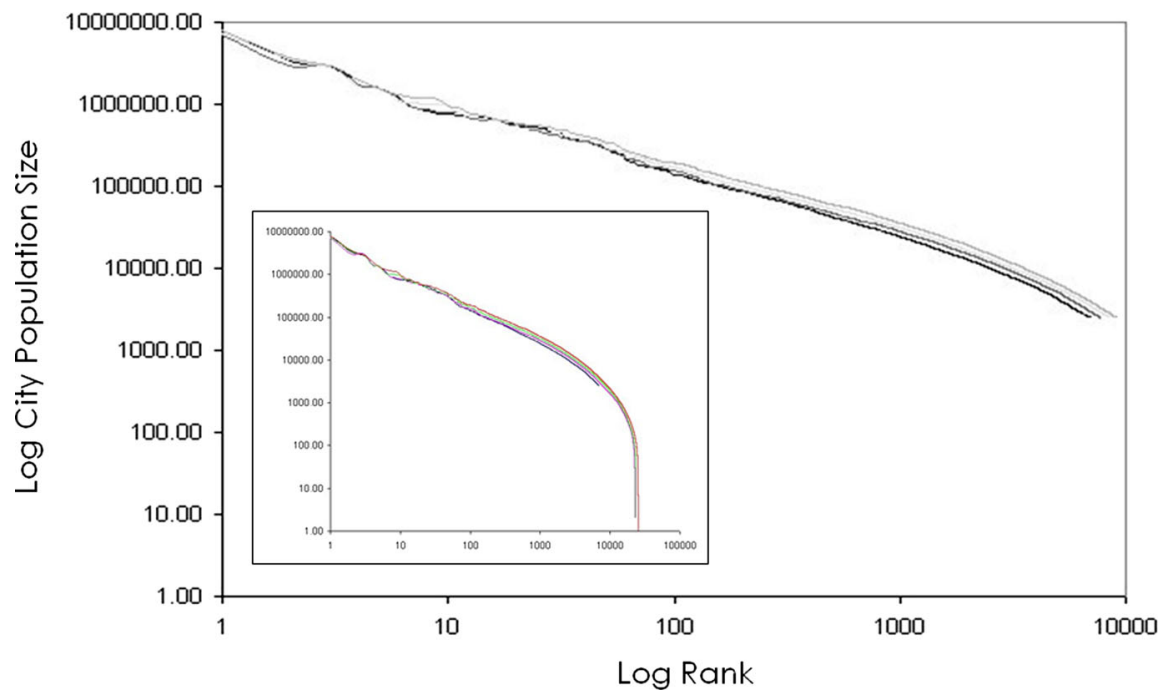

Fig. 4 Rank-size of US city populations 1970-2000 from the populations of incorporated places

The other feature of this data is that when we estimate the rank-size equation, we get values of the scaling coefficient $\beta$ close to unity, which is the theoretical value derived from assumptions about the dynamics of stochastic growth approximating a steady state. This gives some weight to the argument that the US urban system is indeed near to some equilibrium that reflects growth from the bottom up as articulated in a variety of theories such as that proposed in (Gabaix 1999). The scaling values range from 0.986 in $1970,0.982$ in 1980, 0.995 in 1990 to 1.014 in 2000 , with $R^{2}$ goodness of fit values from 0.969 to 0.979 . These are remarkable results for those who believe in Zipf's (1949) original hypothesis that the rank-size rule for cities is a pure form. This means that the size of any city of rank $r$ is equal to the size of the population of the next ranked city $r+1$ divided by rank $r$, that is $P_{r}=P_{r+1} / r$, which in terms of our rank-size equation is $P_{r} \sim r^{-1}$. In fact, we have managed to show that for a system of cities at a lower level of spatial scale than the global, we have derived larger scaling coefficient values; this implies greater competition in the terms we have set here. To an extent, this simply indicates that our hypothesis of competition between places getting less as spatial scales get finer remains speculation, which will be confirmed or otherwise as more theories and empirical applications taking these ideas forward are developed.

At first sight, these results suggest that the urban system in the United States is unchanging, in that the most obvious explanation for a system of objects whose signatures remain largely unchanged from decade to decade is that the objects that comprise this are unvarying in their size and position. But in fact the great paradox of social systems such as cities is that they are continually changing in size and position. This has always been the case and is enshrined in an age-old quote from Herodotus in The Histories: he says "I will [tell] the story as I go along of small cities no less than of great. Most of those which were great once are small today; 
Fig. 5 Volatility in city size and position between 1940 and 2000 for US city size data

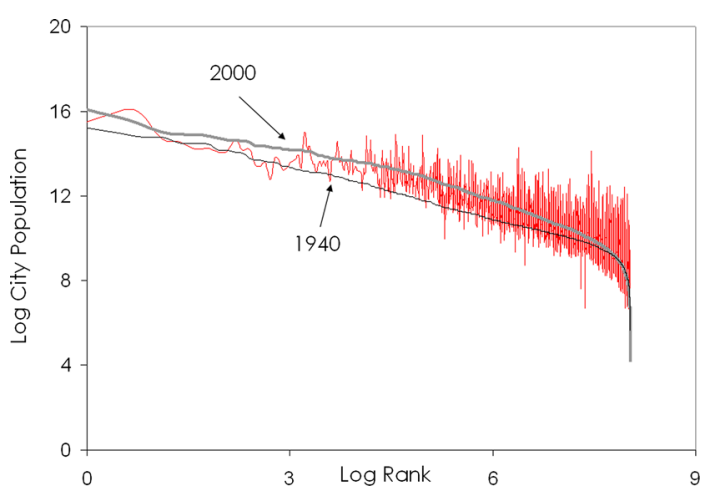

and those which in my own lifetime have grown to greatness, were small enough in the old days" (quoted in Jacobs 1969, frontispiece). We can demonstrate how cities move up and down in the hierarchy quite easily if we take the ranks and populations of cities at one year and compare these same cities with respect to their population at another year but using the ranks from the first year. In Fig. 5, we take data from a set of cities in the US at 1940 and 1990, plot their ranks and sizes on a log plot in the usual way and then produce a new rank plot where we use 1940 ranks for 2000 city populations. This produces an overlay plot which reveals quite considerable volatility in position and size particularly as cities get smaller in size and larger in rank.

The volatility which increases as cities get smaller is entirely consistent with what we know about the creation and destruction of cities. The greatest variations in city size in terms of the growth rates are at the bottom of the urban hierarchy; this is clearly demonstrated by the fact that the differences get smaller (although the log scale irons these out) as the rank falls in Fig. 5.

There are many ways of showing this kind of dynamics and we will digress slightly to outline these. It is entirely possible to extend the idea of Fig. 5 to plot the trajectories of individual cities on the rank-size plot, in other words, we can take the population and rank of an individual city and plot its trajectory over time in this space. In Fig. 6a, we do this for several cities from the top 100 cities database produced from 1790 to the most recent US census, choosing some cities that really do break the mould by either entering or leaving the top 100 or spiralling around in unusual patterns. We can make this a little simpler by getting rid of population size (for rank is to an extent a transform of size) and plotting these trajectories on a clock which is configured where the entire circular cycle is matched to its period. From 1790 to 2000, for example, this rank clock is a temporal circle where rank is plotted on the angular axis and time on the circumference. We show the same data in Fig. 6b.

The stability of the rank-size equations for the aggregate city-size relations as estimated from Eq. (4) is shown by small variations in the scaling parameters which we have estimated for all time periods from 1790 in ten-year intervals until 2000. These are for the top 100 cities over this 210-year time period, noting however that the first date at which 100 cities were recorded in the US Census was 1840. The 

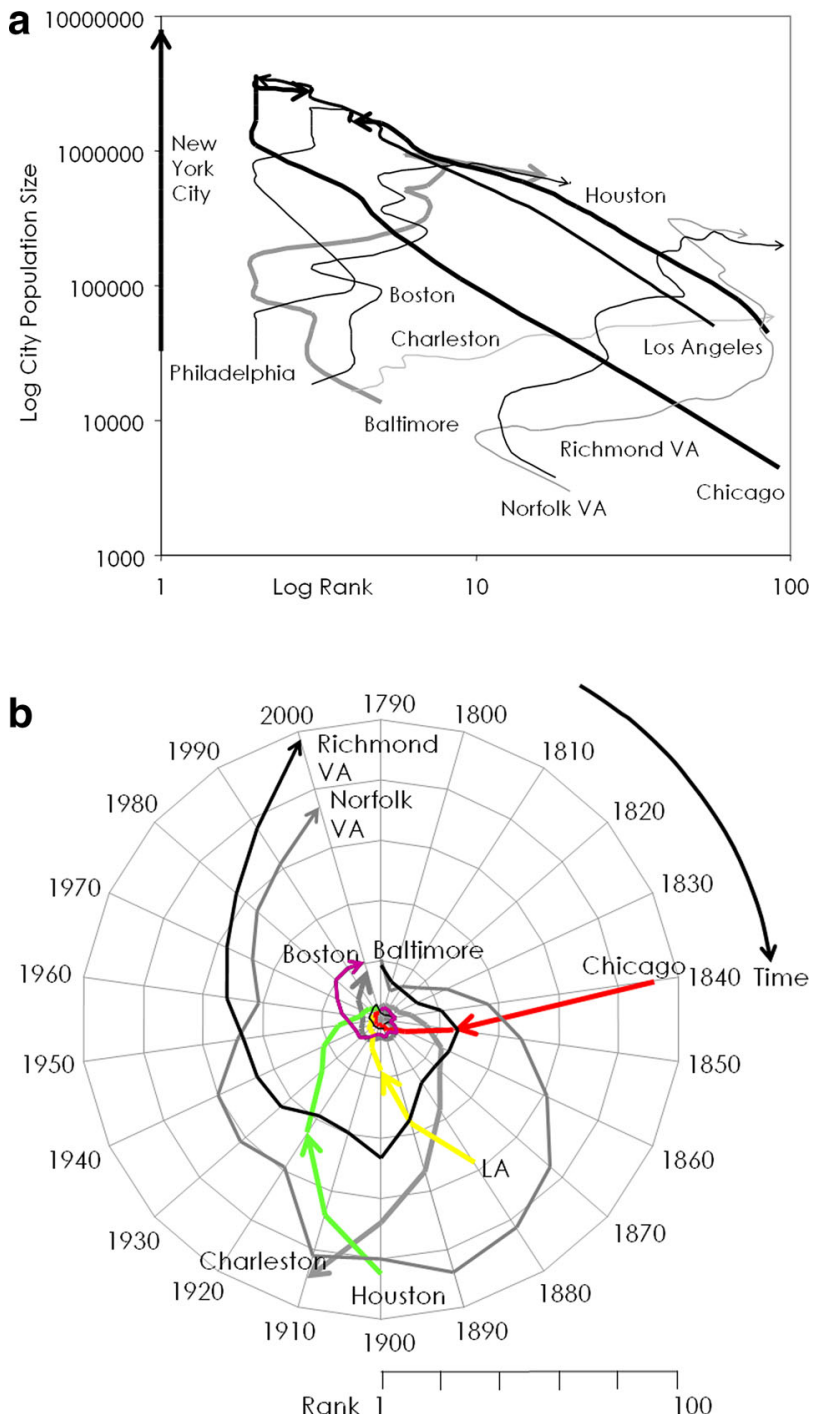

Fig. 6 The dynamics of rank and size using: a rank space (left), and b rank clock (right)

average over this period is $\beta=0.888$ with a standard deviation of 0.069 , which reflects the narrow range of values from 0.737 to 0.990 . We have various related measures of volatility and the reader is referred to other papers for this detail (Batty 2015).

Our last visualisation of this paradox of stability and volatility pertains to the changes in the population of the primary urban areas in Britain, where we examine the rank-size relations of the sixty-three cities $^{1}$ that comprise these urban areas at

\footnotetext{
1 These urban areas, called primary urban areas (PUSs) are shown at http://www.centreforcities.org/puas/.
} 


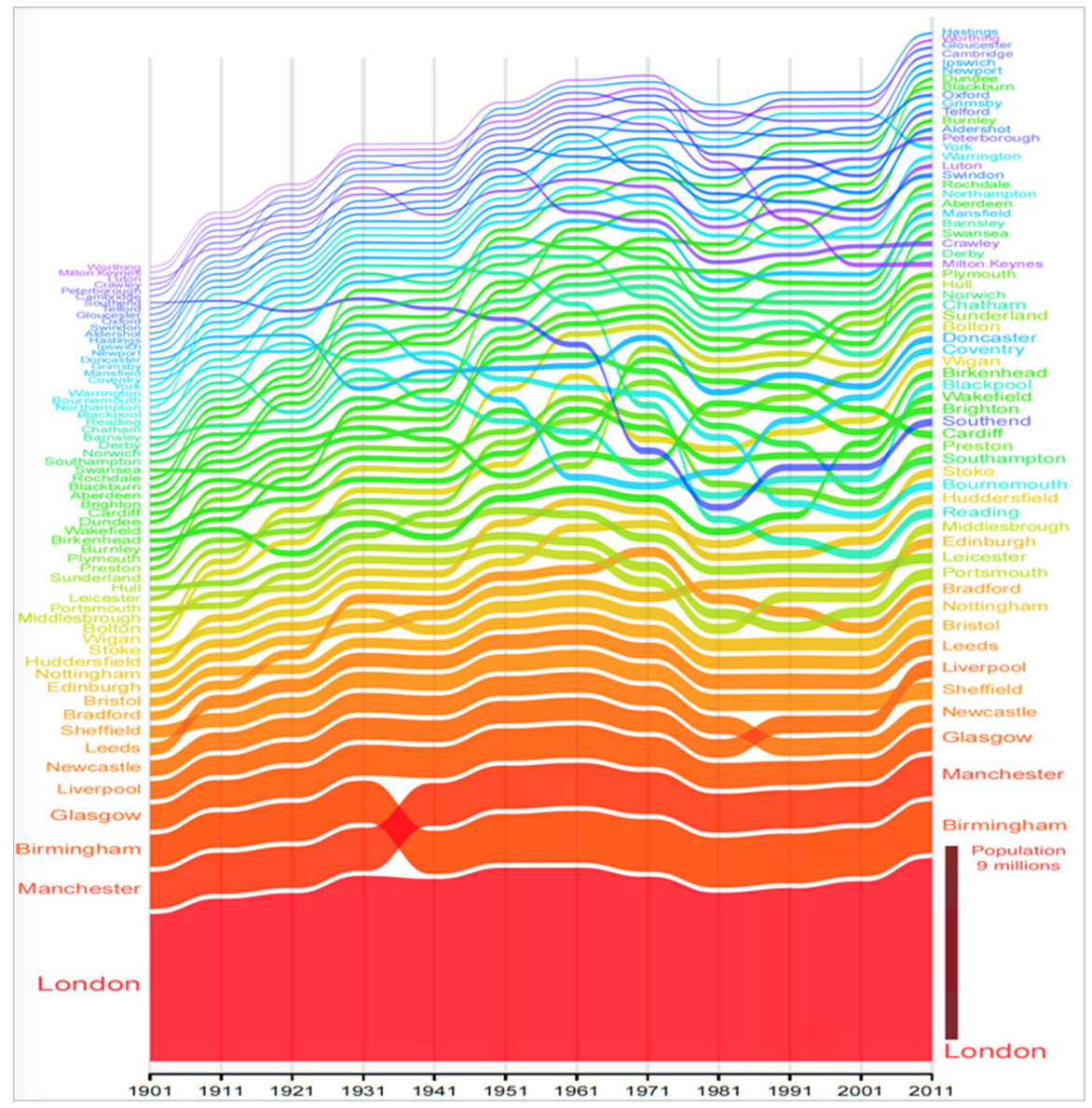

Fig. 7 Rank-Size Trajectories for the Evolution of the British Urban System from 1901 to 2011

each decade from 1901 to 2011. In Fig. 7 we show another graphic which incorporates the size and rank together. Rank is on the vertical axis and time on the horizontal, which is the similar to the rank clock in that these two variables have distinct geometric positions. But this time, we plot the change in rank over time by stacking the rank trajectories according to their size, which is reflected in their width. What is quite clear is the smaller towns of lower rank change in their ranking much more than the largest cities. However, as the largest cities take more of the graph, when they do change this is significant: for example Manchester loses its second position to Birmingham midway through the twentieth century, while a small town such as Milton Keynes rises dramatically through the rankings from mid-century onwards. Each trajectory is also coloured according the ranks at the first time period, which helps visualise their movement as they change through time. 


\section{Neighbourhoods in Cities}

As we move to finer scales, the division of the city into small areas or neighbourhoods becomes increasingly arbitrary. To an extent, the way we define these units is based on administrative considerations rather than natural units that pertain to the landscape of physical topography, although in fact administrative
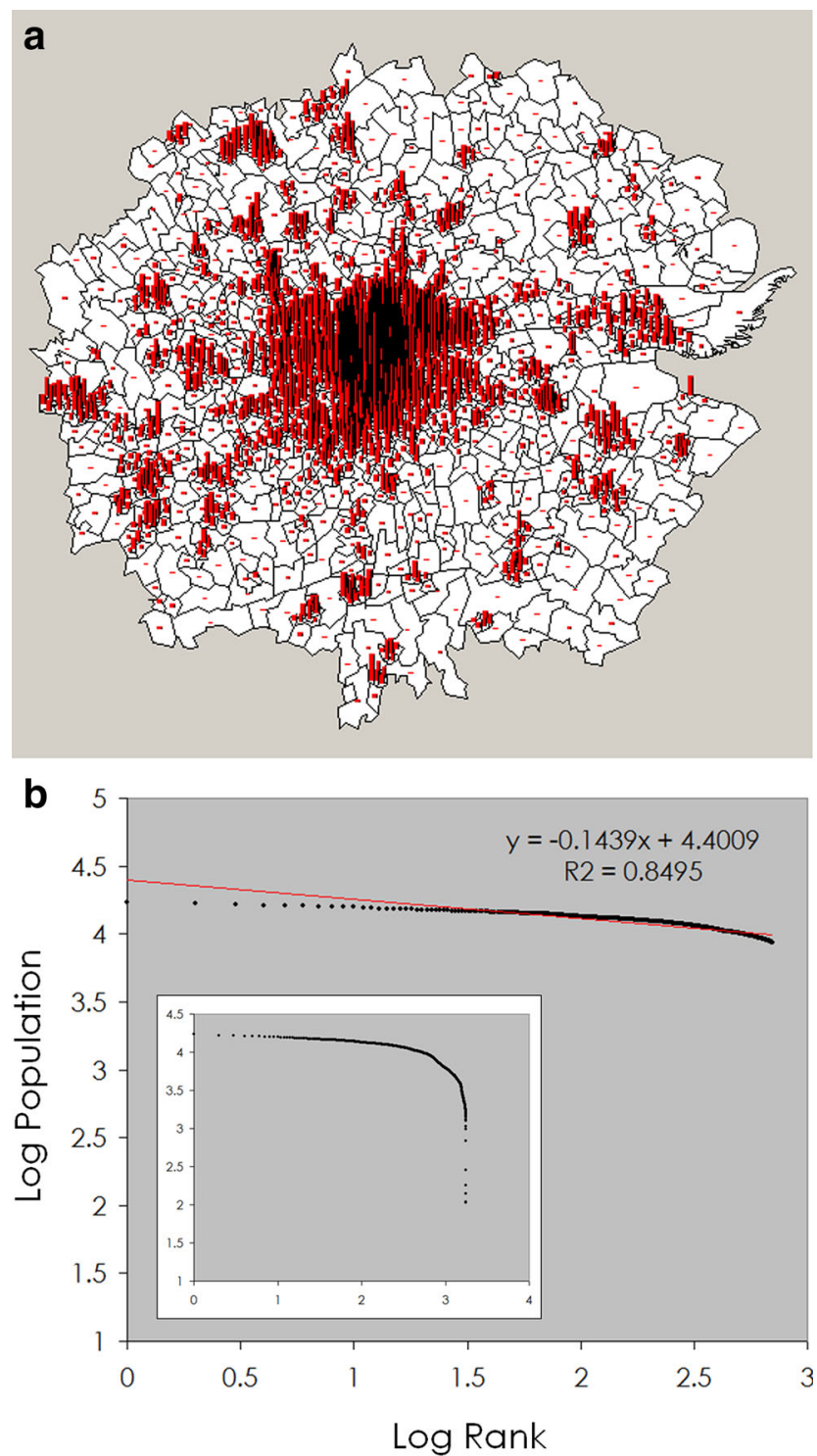

Fig. 8 Scaling of populations in the spatial units comprising the London region. a Histograms of population sizes (left); b Rank-size plots of log linear populations (right) 
units often reflect such considerations as well as issues pertaining to electoral registration, population size, land area, and the way services are delivered to their populations. In this sense, we would expect there to be less differentiation by size but even more volatility amongst smaller units, particularly in city systems which are growing and expanding around their peripheries. We will not examine this volatility here, but a measure of the relative competition between units is reflected in the rank-size distributions which we show for London and its outer metropolitan area at 2001, where the units are based on 1,767 'wards', electoral districts at the lowest level which councillors represent. In Fig. 8a, we show the populations of these wards by histogram across the London region, which is a schematic showing the polycentric nature of the region and the fact that a small number of units have the largest populations; in Fig. $8 \mathrm{~b}$ we show the logarithmic rank-size distribution of these populations.

The graphs in Fig. 8b show that characteristic signature of size is lognormal and when we cut off the distribution by simply leaving its heavy tail—we do this by simply examining the graph visually and taking the top 700 population sizes-we get a linear regression which generates a low scaling coefficient of 0.144 . In fact, from Fig. $8 \mathrm{~b}$ the slope seems actually somewhat flatter and the correlation with the linear fit is not as high as in previous examples. If we then fit the line to the top 100 cities, which is the real heavy tail, then we improve the $R^{2}$ statistic dramatically to 0.971 and the coefficient falls to 0.056 , very different from the assumed pure Zipf Law, which we demonstrated for US cities, yields a coefficient of near 1 . In passing we might say that generating regression coefficients of greater than 1 in fitting city size distributions is unusual (Cristelli et al. 2012), although our notion that this reflects the upper limit of a purely competitive system is entirely speculative.

\section{Buildings: Scaling Skyscrapers}

The biggest problem in deriving properties of scaling for any system of spatial objects involves the completeness of the set. Indeed the very notion that we might approximate what appear to be lognormally distributed sets by power laws which fit their long or heavy tail of their frequencies is a recognition that there is often incompleteness in the set of objects anyway. The fact that we fit power functions to a small subset of the overall set is based on the assumption that the biggest objects in the set are most important. But as is clear from the fact that members of the set change position all the time, the biggest objects may not all remain so and thus this assumption is contestable. Moreover, all the action in systems of this kindparticularly those that evolve through growth and change-occurs in the smallest objects, which contain the dynamics to grow to bigness or decline to oblivion. There are very few examples of where a complete set has been handled in terms of scaling relations. In fact using data from 3D models where heights of building blocks are produced from remote sensed data ${ }^{2}$ does give complete sets, as we have used in a previous analysis of building allometry in Greater London (Batty et al. 2008), but the problem with this data (and indeed that for many scales of city populations) is

\footnotetext{
${ }^{2}$ From LIDAR data: LIght $\underline{\text { Detection }}$ And $\underline{\text { Ranging data. }}$
} 
that the overall orders of magnitude available for these systems is quite narrow. For scaling relations to be validated as measures of self-similarity, we require wider ranges of size: in fact this is not possible in terms of many of these kinds of system.

When we move to the scale of buildings, we cross a boundary in interpretation where we no longer speak of buildings growing or declining, at least in terms of their continuity of change. Buildings are by and large 'designed' in the modern world and although it may be possible to see more natural trends in their evolution if they are grouped into classes and different locations, we will in fact examine actual buildings on their individual sites and explore the consequences of scaling for such sets. In fact there are other properties of buildings with respect to their size and shape that can be measured and compared in terms of their distribution, such as volume, wall area and plan depth (Steadman et al. 2011) and the edges of their outlines (Mohajeri and Gudmundsson 2014). Here we use height, for we are able to examine buildings by their actual size in terms of floor space, height, number of stories and so on. The best data sets available in the public domain are those for high buildings; an immediately available set of all those greater than 150 metres in height can be extracted from The Skyscraper Center's Global Tall Building Database which is run by the CTBUH (Council on Tall Buildings and Urban Habitat). The basic data consists of all such buildings completed, under construction, and proposed, including those that have been demolished. We have only included in fact those completed and under construction but the data in question dates from 1909 to a final construction date of 2020 . There are 4325 buildings in this database but the fact that we have chosen 150 metres as the lower level cut-off means that many of the highest buildings have been completed or under construction in the last two decades. In fact, the average year of construction of the top ten buildings in the set is 2016 while that of the lowest ten is 2007 . The average height of the total set is 198 metres; only $12 \%$ of the set are greater than $250 \mathrm{~m}$.

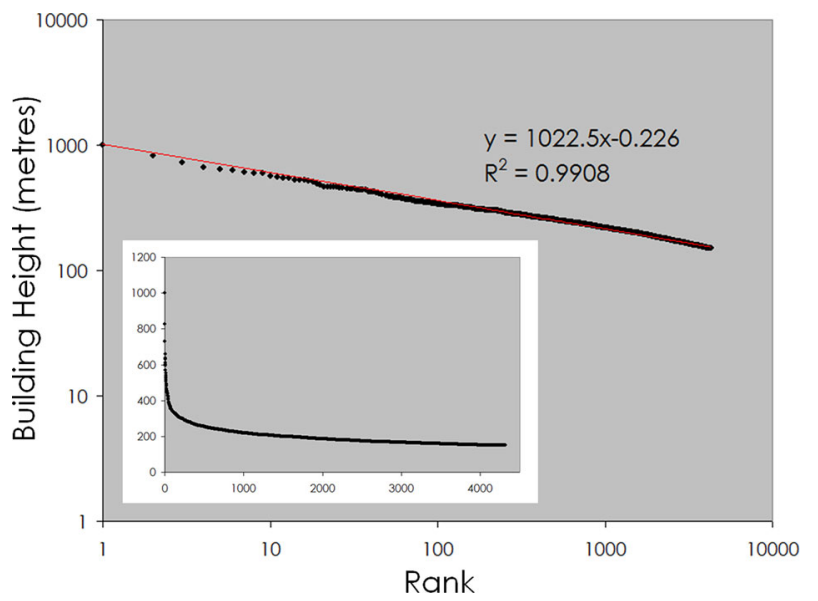

Fig. 9 Perfect scaling? The highest buildings (skyscrapers) from the CTHUB database 
Our first demonstration of the scaling inherent in this high building data is shown in Fig. 9, where it is clear that the scaling is very strong in terms of the proportion of the variance exchanged and there is little sense of the fact that the distribution is lognormal.

As the data contains several building attributes such as name, location by city, height (in metres and feet), floors completed, materials used, and land use activity, we can partition it in many ways, by city, by nation or region or continental block, or by year of construction. What we have done is to divide the data into five decades of construction: 1909-1960 (which includes the high building boom of the late

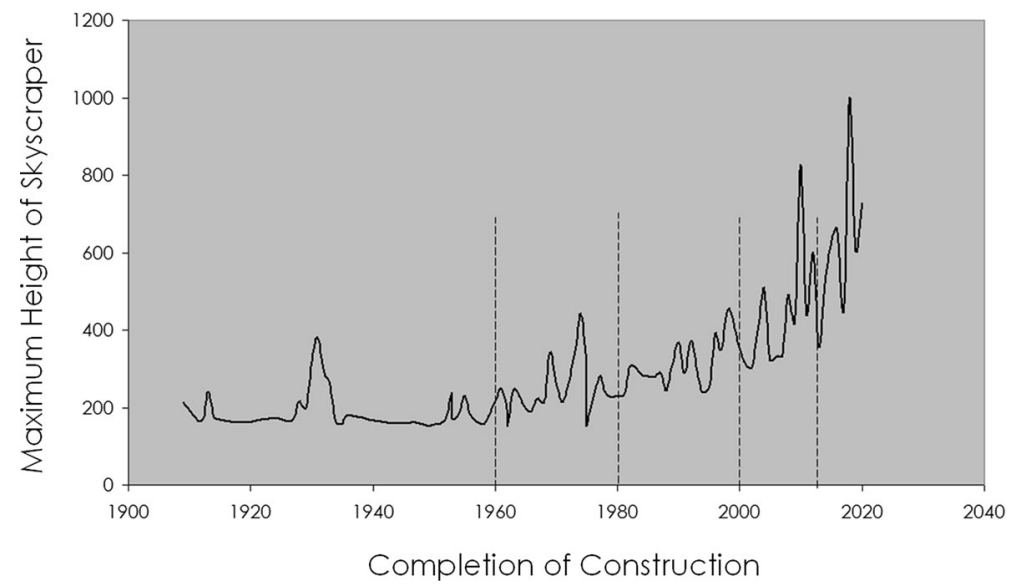

Fig. 10 The temporal profile of high building heights showing the maximum height per year
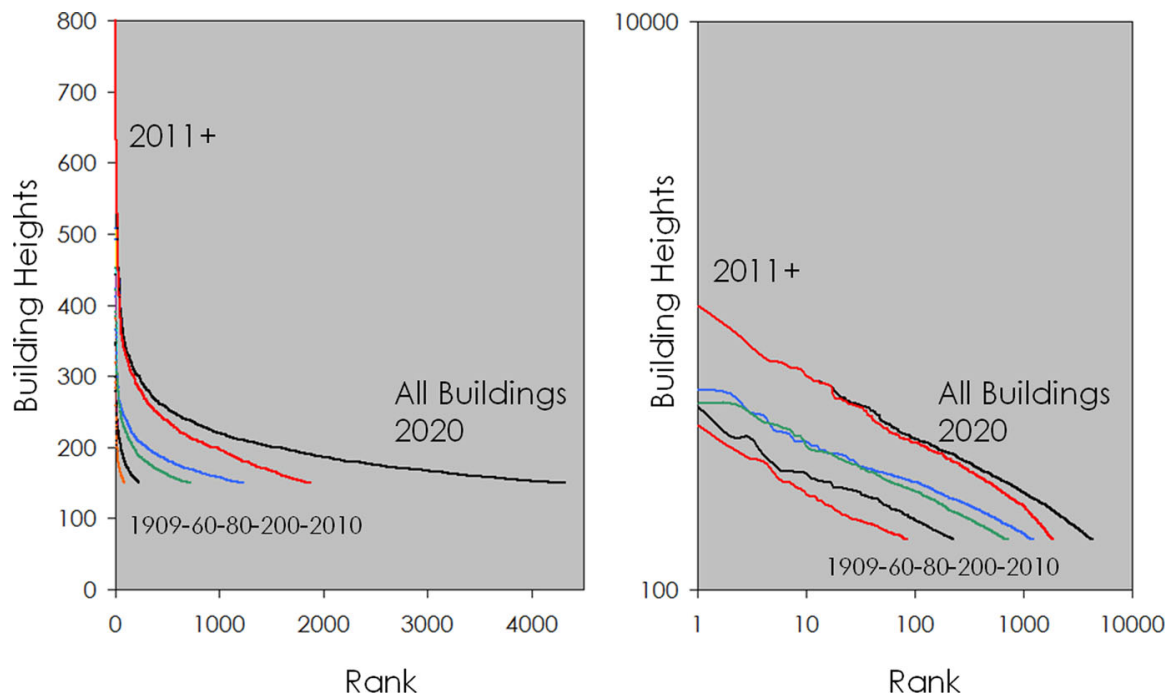

Fig. 11 Rank-size distributions for high buildings across time 
Table 1 Scaling coefficients for high buildings constructed during temporal periods from 1909

\begin{tabular}{llll}
\hline Period & Numbers of buildings & $R^{2}$ & Scaling parameter $\beta$ \\
\hline All at 2020 & 4325 & 0.991 & 0.226 \\
$2011+$ & 1877 & 0.975 & 0.257 \\
$2001-2010$ & 1232 & 0.989 & 0.173 \\
$1981-2000$ & 718 & 0.996 & 0.188 \\
$1961-1980$ & 225 & 0.989 & 0.177 \\
$1909-1960$ & 85 & 0.983 & 0.193 \\
\hline
\end{tabular}

* Note that the period numbers do not sum to 4325 , as in 188 cases, the data of construction is missing

1920s, early 1930s), 1961-1980, 1981-2000, 2001-2010, and 2011+ onwards. We show the activity of construction and completion for these periods in Fig. 10 and it is clear that in general there is a progressive rise in the height of tall buildings over the last century. The average heights (metres) per period associated with this data are: 180 in 1909-1960; 183 in 1961-1980; 186 in 1981-2000; 187 in 2001-2010; and 218 in 2011+. We have fitted rank-size relations to these distributions for each of these periods and we find that the scaling coefficients do not change much systematically over the last century, More recently the coefficients seem to be increasing a little but we do not regard any of this as significant. Thus there is no real change in the relative slope of the rank-size curves which we show in Fig. 11 and in Table 1 for these different periods.

The volatility in this data is of a very different nature to that for city size distributions in that, as we have excluded demolitions and additions to skyscraper height (of which there are very few in the database), buildings cannot rise in rank. As ever higher buildings are built, buildings can only ever fall in rank and if we were to plot a rank clock or examine these in the rank space or even use the timespace-size graph (as in Fig. 7), the buildings would spiral out of the picture or down the graph as higher buildings take their place. The patterns that such spirals generate define the degree of change. For further explorations of these, readers are referred to related work (Batty 2013, 2015).

\section{Implications for Architecture, Planning and Our Understanding of Cities}

A major consequence which leads to scaling in many social and physical systems is that such systems evolve, develop from the bottom up. In terms of populations and buildings that define cities at different scales, cities are small before they become big and this means that they grow or decline incrementally, usually through additions or demolitions or migrations of the basic components that compose their form and function. Although this argument has gained considerable momentum recently with the rise of complexity theory in many sciences, it has been implicit in architecture and city planning for many years (Jacobs 1961). The tension between organically growing cities and their architecture and the top-down imposed nature 
of much city planning and contemporary architecture points up this tension. It behoves us to ask the question: if properties of naturally and artificially evolving systems such as cities, display such regularities as scaling, to what extent can design and intervention manipulate such signatures and fight against them with respect to what we might define as more appropriate forms and functions?

To an extent, there are some who argue that scaling represents optimal design, as it is also the signature of nature, of allometry and in this sense, represents ways of generating sustainable forms and functions (Carlson and Doyle 2000). This sentiment needs to be explored in much more detail, for it is crucial to the nature of design and policy analysis in cities at all scales. Kelvin's dictum that we need to measure it to have confidence in its meaning is essential if we are ever to move beyond mere rhetoric and analogy in a field where quite clearly a scientific approach has great promise. In this paper, all we have done is catalogue a variety of applications of the scaling law, noting how stability and volatility are essential properties of the systems that give rise to such laws. What we now need are dynamic models that examine the interactions between the objects or events and specify how the dynamics of these interactions affect the ultimate distribution of the objects. It is very likely that, rather than by interfering with the size of the objects per se through design, we may be able to modify interactions that in turn condition the way policy and planning is implemented from the top down (as well as bottom up). Considerably more work needs to be done, not just with these simple systems but on the whole range of systems which are formed from competing components and their evolution. The nature of the competitive process, the interactions that keep the system stable but also might change to ensure a degree of volatility for adaptation, and the ways in which objects are bounded in scope, merge, coalesce and dissolve, are important issues in our further understanding of this kind of spatial complexity.

\section{References}

Batty, M. 2006. Rank Clocks. Nature 444: 592-596.

Batty, M. 2013. The New Science of Cities. Cambridge MA: The MIT Press.

Batty, M. 2015. Scale, Power Laws, and Rank-size in Spatial Analysis, in C. Brunsdon, A. Singleton. Editors. Geocomputation: A Practical Primer. London: Sage. 40-60.

Batty, M., R. Carvalho, A. Hudson-Smith, R. Milton, D. Smith, and P. Steadman. 2008. Scaling and Allometry in the Building Geometries of Greater London. European Physical Journal B 63: 303-318.

Blank, A., and S. Solomon. 2000. Power Laws in Cities Population, Financial Markets and Internet Sites: Scaling in Systems with a Variable Number of Components. Physica A 287: 279-288.

Carlson, J. M., and J. Doyle. 2000. Power Laws, Highly Optimized Tolerance and Generalized Source Coding. Physical Review Letters 8424: 5656-5659.

Clauset, C., C. R. Shalizi, and M. E. J. Newman. 2009. Power-Law Distributions in Empirical Data. SIAM Review 51: 661-703.

Cristelli, M., M. Batty, and L. Pietronero. 2012. There is More than a Power Law in Zipf. Scientific Reports. 6 November 2012, at www.nature.com/srep/

Gabaix, X. 1999. Zipf's Law for Cities: An Explanation. The Quarterly Journal of Economics 114: 739-767.

Jacobs, J. 1961. The Death and Life of Great American Cities. New York: Random House.

Jacobs, J. 1969. The Economy of Cities. New York: Vintage Books. 
March, L. 2011. Forty Years of Shape and Shape Grammars, 1971-2011. Nexus Network Journal 13: $5-13$.

Mohajeri, N., and A. Gudmundsson. 2014. Quantifying the Differences in Geometry and Size Distributions of Buildings Within Cities. Nexus Network Journal 16: 417-436.

Newman, M. E. J. 2005. Power Laws, Pareto Distributions and Zipf's Law. Contemporary Physics 46: $323-351$.

Pisarenko, V. F. and Sornette, D. 2012. Robust Statistical Tests of Dragon-Kings beyond Power Law Distributions. European Physical Journal Special Topics 205: 95-115.

Schumpeter, J. A. 1939. Business Cycles. New York: McGraw-Hill Book Company.

Simon, H. A. 1955. On a Class of Skew Distribution Functions, Biometrika 42, 425-440.

Steadman, P., Evans, S. and Batty, M. 2011. Wall Area, Volume and Plan Depth in the Building Stock. Building Research and Information 37: 455-467.

Zipf, G. K. 1949. Human Behavior and the Principle of Least Effort. Reading MA: Addison-Wesley.

Michael Batty is Bartlett Professor of Planning at University College London where he is Chair of the Centre for Advanced Spatial Analysis (CASA). He has worked on computer models of cities and their visualisation since the 1970s and has published several books, such as Cities and Complexity (MIT Press, 2005) which won the Alonso Prize of the Regional Science Association in 2011, and most recently The New Science of Cities (MIT Press, 2013). His blog www.complexcity.info covers the science underpinning the technology of cities and his posts and lectures on big data and smart cities are at www. spatialcomplexity.info. His research group is working on simulating long term structural change and dynamics in cities as well as their visualisation. He is a Fellow of the British Academy (FBA) and the Royal Society (FRS), and was awarded the CBE in the Queen's Birthday Honours in 2004 